\title{
A Representation of Lattice Effect Algebras by Means of Right Near Semirings with Involution
}

\author{
Ivan Chajda ${ }^{1} \cdot$ Helmut Länger ${ }^{2}(D)$
}

Received: 8 August 2016 / Accepted: 11 November 2016 / Published online: 25 November 2016

(C) The Author(s) 2016. This article is published with open access at Springerlink.com

\begin{abstract}
Since every lattice effect algebra decomposes into blocks which are MV-algebras and since every MV-algebra can be represented by a certain semiring with an antitone involution as shown by Belluce, Di Nola and Ferraioli, the natural question arises if a lattice effect algebra can also be represented by means of a semiring-like structure. This question is answered in the present paper by establishing a one-to-one correspondence between lattice effect algebras and certain right near semirings with an antitone involution.
\end{abstract}

Keywords Effect algebra $\cdot$ Lattice effect algebra $\cdot$ Right near semiring $\cdot$ Antitone involution $\cdot$ Effect near semiring

Effect algebras were introduced by Foulis and Bennett [8] in order to axiomatize unsharp logics of quantum mechanics. Although the definition of an effect algebra looks elementary, these algebras have several very surprising properties. Concerning these properties the reader is referred to the monograph [7] by Dvurečenskij and Pulmannová. In particular, every effect algebra induces a natural partial order relation and thus can be considered as a bounded poset. If this poset is a lattice, the effect algebra is called a lattice effect algebra.

Support of the research by the Austrian Science Fund (FWF), project I 1923-N25, and the Czech Science Foundation (GAČR), project 15-34697L, is gratefully acknowledged.

\section{Helmut Länger}

helmut.laenger@tuwien.ac.at

Ivan Chajda

ivan.chajda@upol.cz

1 Faculty of Science, Department of Algebra and Geometry, Palacký University Olomouc, 17. listopadu 12, 77146 Olomouc, Czech Republic

2 Faculty of Mathematics and Geoinformation, Institute of Discrete Mathematics and Geometry, TU Wien, Wiedner Hauptstraße 8-10, 1040 Vienna, Austria 
A representation of lattice effect algebras by means of so-called basic algebras was derived in [2].

It was proved by Riečanová that every lattice effect algebra is built up by blocks which are MV-algebras. A representation of MV-algebras by means of semiring-like structures, so-called MV-semirings, was already published by Belluce et al. in [1] and it was further developed by Di Nola and Russo in [6]. These facts encouraged us to try a similar approach also for lattice effect algebras. The motivation for such a representation by means of semiring-like structures is practically the same as in [1]. The fact that the binary operation in effect algebras is only partial and that one cannot suppose that, whenever extended to a total operation, it would remain commutative and associative, motivated us to use so-called right near semirings instead of semirings. The concept of a right near semiring was introduced by the authors in [3]. These algebras seem to be an appropriate tool when equipped with an involution. Similarly as in [1], we ask them to satisfy six more natural axioms. We will call such algebras effect near semirings.

In [5], lattice effect algebras were represented by couples of right near semirings connected by a certain mapping. In this paper we present a representation of lattice effect algebras by means of a single right near semiring which is equipped with an involution. It turns out that this representation is one-to-one.

We start with the definition of an effect algebra.

Definition 1 An effect algebra is a partial algebra $\mathbf{E}=(E, \oplus, 0,1)$ of type $(2,0,0)$ satisfying conditions (E1) - (E4) for all $x, y, z \in E$ :

(E1) If $x \oplus y$ exists, so does $y \oplus x$ and $x \oplus y=y \oplus x$;

(E2) if $x \oplus y$ and $(x \oplus y) \oplus z$ exist, so do $y \oplus z$ and $x \oplus(y \oplus z)$ and $(x \oplus y) \oplus z=x \oplus(y \oplus z)$;

(E3) there exists a unique $x^{\prime} \in E$ such that $x \oplus x^{\prime}$ is defined and $x \oplus x^{\prime}=1$;

(E4) If $x \oplus 1$ exists then $x=0$.

Since ' is a unary operation on $E$ it can be regarded as a further fundamental operation. Hence in the following we will write $\mathbf{E}=\left(E, \oplus,^{\prime}, 0,1\right)$ instead of $\mathbf{E}=(E, \oplus, 0,1)$.

Let $\mathbf{E}=\left(E, \oplus,^{\prime}, 0,1\right)$ be an effect algebra and $a, b \in E$. The following facts are well-known:

(F1) By defining $a \leq b$ if there exists some $c \in E$ such that $a \oplus c$ exists and $a \oplus c=b$, $\left(E, \leq,^{\prime}, 0,1\right)$ becomes a bounded poset with an antitone involution. We call $\leq$ the induced order of $\mathbf{E}$. $\mathbf{E}$ is called a lattice effect algebra if $(E, \leq)$ is a lattice.

(F2) $a \oplus b$ exists if and only if $a \leq b^{\prime}$.

(F3) $\quad a \oplus 0$ and $0 \oplus a$ exist and $a \oplus 0=0 \oplus a=a$.

(F4) $\quad\left(a^{\prime}\right)^{\prime}=a$

We recall Proposition 1.8.6 from [7]:

Proposition 2 Let $\mathbf{E}=\left(E, \oplus,{ }^{\prime}, 0,1\right)$ be a lattice effect algebra, $\vee$ and $\wedge$ denote its lattice operations and $a, b, c \in E$. If $a \oplus c$ and $b \oplus c$ exist then $(a \wedge b) \oplus c=(a \oplus c) \wedge(b \oplus c)$.

The concept of a right near semiring was introduced by the authors in [3] as follows (cf. also [4] and [5]):

Definition 3 A right near semiring is an algebra $\mathbf{R}=(R,+, \cdot, 0,1)$ of type $(2,2,0,0)$ satisfying conditions (R1) - (R4): 
(R1) $(R,+, 0)$ is a commutative monoid;

(R2) $(R, \cdot, 1)$ is a groupoid with neutral element 1 ;

(R3) $(x+y) z \approx x z+y z$;

(R4) $x 0 \approx 0 x \approx 0$.

$\mathbf{R}$ is called idempotent if it satisfies the identity $x+x \approx x$. In this case $(R,+)$ is a semilattice which we will consider as a join-semilattice whose partial order relation $\leq$ will be called the induced order of $\mathbf{R}$. Observe that then $x \leq y$ is equivalent to $x+y=y$ and that 0 is the least element of $(R, \leq)$.

Now we define right near semirings with involution.

Definition 4 A right near semiring with involution is an algebra $\mathbf{R}=\left(R,+, \cdot,{ }^{\prime}, 0,1\right)$ of type $(2,2,1,0,0)$ such that its reduct $\mathbf{R}_{1}=(R,+, \cdot, 0,1)$ is an idempotent right near semiring and ' is an antitone involution on $(R, \leq)$ where $\leq$ denotes the induced order of $\mathbf{R}_{1}$ which we will call the induced order of $\mathbf{R}$, too.

Remark 5 The element 1 need not be the greatest element of $(R, \leq)$ which is $0^{\prime}$. If one defines $x+{ }^{\prime} y:=\left(x^{\prime}+y^{\prime}\right)^{\prime}$ for all $x, y \in R$ then $\left(R,+,+^{\prime},{ }^{\prime}, 0,0^{\prime}\right)$ is a bounded lattice with an antitone involution where + denotes the supremum operation and $+^{\prime}$ the infimum operation.

Now we define our counterpart of lattice effect algebras.

Definition 6 An effect near semiring is a right near semiring $\mathbf{R}=\left(R,+, \cdot,{ }^{\prime}, 0,1\right)$ with involution satisfying conditions (N1) - (N6) for all $x, y \in R$ :

(N1) $0^{\prime}=1$;

(N2) $x \leq y$ if and only if $x y^{\prime}=0$;

(N3) If $x^{\prime} y^{\prime}=0$ then $x y=y x$;

(N4) If $x^{\prime} y^{\prime}=0$ and $(x y)^{\prime} z^{\prime}=0$ then $y^{\prime} z^{\prime}=0, x^{\prime}(y z)^{\prime}=0$ and $(x y) z=x(y z)$;

(N5) If $x y=0$ then there exists an element $z$ of $R$ with $x z=0$ and $x^{\prime} z^{\prime}=y$;

(N6) $x\left(y x^{\prime}\right)=0$.

The following theorem shows that to every lattice effect algebra there can be assigned an effect near semiring in some natural way.

Theorem 7 Let $\mathbf{E}=\left(E, \oplus,{ }^{\prime}, 0,1\right)$ be a lattice effect algebra with lattice operations $\vee$ and $\wedge$ and put

$$
x y:=\left(\left(x^{\prime} \wedge y\right) \oplus y^{\prime}\right)^{\prime}
$$

for all $x, y \in E$. Then $x y$ is well-defined because of $x^{\prime} \wedge y \leq y$ and, moreover, $\mathbb{R}(\mathbf{E}):=$ $\left(E, \vee, \cdot,{ }^{\prime}, 0,1\right)$ is an effect near semiring.

Proof Let $a, b, c \in E .(E, \vee, 0)$ is an idempotent commutative monoid. Since

$$
\begin{aligned}
& a 1=\left(\left(a^{\prime} \wedge 1\right) \oplus 1^{\prime}\right)^{\prime}=\left(a^{\prime} \oplus 0\right)^{\prime}=\left(a^{\prime}\right)^{\prime}=a \text { and } \\
& 1 a=\left(\left(1^{\prime} \wedge a\right) \oplus a^{\prime}\right)^{\prime}=\left((0 \wedge a) \oplus a^{\prime}\right)^{\prime}=\left(0 \oplus a^{\prime}\right)^{\prime}=\left(a^{\prime}\right)^{\prime}=a,
\end{aligned}
$$


$(E, \cdot, 1)$ is a groupoid with neutral element. Moreover, using Proposition 2 we compute

$$
\begin{aligned}
(a \vee b) c & =\left(\left((a \vee b)^{\prime} \wedge c\right) \oplus c^{\prime}\right)^{\prime}=\left(\left(a^{\prime} \wedge b^{\prime} \wedge c\right) \oplus c^{\prime}\right)^{\prime}=\left(\left(\left(a^{\prime} \wedge c\right) \wedge\left(b^{\prime} \wedge c\right)\right) \oplus c^{\prime}\right)^{\prime}= \\
& =\left(\left(\left(a^{\prime} \wedge c\right) \oplus c^{\prime}\right) \wedge\left(\left(b^{\prime} \wedge c\right) \oplus c^{\prime}\right)\right)^{\prime}=\left(\left(a^{\prime} \wedge c\right) \oplus c^{\prime}\right)^{\prime} \vee\left(\left(b^{\prime} \wedge c\right) \oplus c^{\prime}\right)^{\prime}= \\
& =(a c) \vee(b c) .
\end{aligned}
$$

Clearly,

$$
\begin{aligned}
& a 0=\left(\left(a^{\prime} \wedge 0\right) \oplus 0^{\prime}\right)^{\prime}=(0 \oplus 1)^{\prime}=1^{\prime}=0 \text { and } \\
& 0 a=\left(\left(0^{\prime} \wedge a\right) \oplus a^{\prime}\right)^{\prime}=\left(a \oplus a^{\prime}\right)^{\prime}=1^{\prime}=0 .
\end{aligned}
$$

Further, $a \leq b$ implies $b^{\prime} \leq a^{\prime}$, and we have $\left(a^{\prime}\right)^{\prime}=a$. From this we conclude that $a \leq b$ is equivalent to $b^{\prime} \leq a^{\prime}$. It remains to prove axioms (N1) - (N6) of Definition 6 .

(N1) $0^{\prime}=1$ holds in $\mathbf{E}$ and hence also in $\mathbb{R}(\mathbf{E})$.

(N2) By (E3) we have that $a^{\prime} \wedge b^{\prime}=b^{\prime}$ implies $b \oplus\left(a^{\prime} \wedge b^{\prime}\right)=b \oplus b^{\prime}=1$ and, conversely, if $b \oplus\left(a^{\prime} \wedge b^{\prime}\right)=1$ then $a^{\prime} \wedge b^{\prime}=b^{\prime}$. Hence the following are equivalent: $a \leq b$, $b^{\prime} \leq a^{\prime}, a^{\prime} \wedge b^{\prime}=b^{\prime}, b \oplus\left(a^{\prime} \wedge b^{\prime}\right)=1,\left(a^{\prime} \wedge b^{\prime}\right) \oplus b=1,\left(\left(a^{\prime} \wedge b^{\prime}\right) \oplus b\right)^{\prime}=0, a b^{\prime}=0$.

(N3) According to (N2) the following are equivalent: $a^{\prime} b^{\prime}=0, a^{\prime} \leq b, a^{\prime} \oplus b^{\prime}$ exists. In this case $a b=\left(\left(a^{\prime} \wedge b\right) \oplus b^{\prime}\right)^{\prime}=\left(a^{\prime} \oplus b^{\prime}\right)^{\prime}$. Hence $a^{\prime} b^{\prime}=0$ implies $a b=\left(a^{\prime} \oplus b^{\prime}\right)^{\prime}=$ $\left(b^{\prime} \oplus a^{\prime}\right)^{\prime}=b a$.

(N4) Assume $a^{\prime} b^{\prime}=0$ and $(a b)^{\prime} c^{\prime}=0$. Then there exists $a^{\prime} \oplus b^{\prime}, a b=\left(a^{\prime} \oplus b^{\prime}\right)^{\prime}$ and there exists $\left(a^{\prime} \oplus b^{\prime}\right) \oplus c^{\prime}$. Hence there exist $b^{\prime} \oplus c^{\prime}$ and $a^{\prime} \oplus\left(b^{\prime} \oplus c^{\prime}\right)$ and $\left(a^{\prime} \oplus b^{\prime}\right) \oplus c^{\prime}=a^{\prime} \oplus\left(b^{\prime} \oplus c^{\prime}\right)$. This shows $b^{\prime} \leq c, b^{\prime} c^{\prime}=0,\left(b^{\prime} \oplus c^{\prime}\right)^{\prime}=b c$ and $a^{\prime}(b c)^{\prime}=0$. Therefore

$$
(a b) c=\left(\left(a^{\prime} \oplus b^{\prime}\right) \oplus c^{\prime}\right)^{\prime}=\left(a^{\prime} \oplus\left(b^{\prime} \oplus c^{\prime}\right)\right)^{\prime}=a(b c) .
$$

(N5) If $a b=0$ then $a \leq b^{\prime}$ according to (N2) and hence according to (F1) and (F2) there exists some $d \in E$ with $a \leq d^{\prime}$ and $a \oplus d=b^{\prime}$ which shows $a d=0$ and $a^{\prime} d^{\prime}=(a \oplus d)^{\prime}=b$.

(N6) We have $a \leq a \oplus\left(b^{\prime} \wedge a^{\prime}\right)=\left(b^{\prime} \wedge a^{\prime}\right) \oplus a=\left(b a^{\prime}\right)^{\prime}$ and hence $a\left(b a^{\prime}\right)=0$ according to $(\mathrm{N} 2)$.

In the next proofs we will need the following result:

Lemma 8 Let $\mathbf{R}=\left(R,+, \cdot,{ }^{\prime}, 0,1\right)$ be an effect near semiring, $\leq$ denote its induced order and $a, b \in R$. Then $a \leq b$ if and only if there exists some $c \in R$ with $a \leq c$ and $a^{\prime} c=b^{\prime}$.

Proof If $a \leq b$ then $a b^{\prime}=0$ according to (N2) and hence there exists some $d \in R$ with $a d=0$ and $a^{\prime} d^{\prime}=b^{\prime}$ according to (N5) whence $a \leq d^{\prime}$ according to (N2). Conversely, assume there exists some $c \in R$ with $a \leq c$ and $a^{\prime} c=b^{\prime}$. Then $a c^{\prime}=0$ according to (N2) which shows $a^{\prime} c=c a^{\prime}$ according to (N3). From this we conclude $b^{\prime}=c a^{\prime}$ and therefore $a b^{\prime}=a\left(c a^{\prime}\right)=0$ according to (N6). This shows $a \leq b$ according to (N2).

Now we show that to every effect near semiring we can assign a lattice effect algebra in some natural way.

Theorem 9 Let $\mathbf{R}=\left(R,+, \cdot,^{\prime}, 0,1\right)$ be an effect near semiring, $\leq$ denote its induced order and for $x, y \in R$ put

$$
x \oplus y:=\left(x^{\prime} y^{\prime}\right)^{\prime}, \text { provided } x \leq y^{\prime} .
$$


Then $\mathbb{E}(\mathbf{R}):=\left(R, \oplus,^{\prime}, 0,1\right)$ is a lattice effect algebra with induced order $\leq$.

Proof Let $a, b, c \in R$.

(E1) Assume $a \oplus b$ exists. Then $a \leq b^{\prime}$ according to the definition of $\oplus$ and hence $a b=0$ according to (N2). Therefore there exists $b \oplus a$ and, according to (N3), $a^{\prime} b^{\prime}=b^{\prime} a^{\prime}$ which implies $a \oplus b=\left(a^{\prime} b^{\prime}\right)^{\prime}=\left(b^{\prime} a^{\prime}\right)^{\prime}=b \oplus a$.

(E2) Assume $a \oplus b$ and $(a \oplus b) \oplus c$ exist. Then $a \leq b^{\prime}$ and $a \oplus b \leq c^{\prime}$ according to the definition of $\oplus$. This implies $a b=0$ and $\left(a^{\prime} b^{\prime}\right)^{\prime} c=0$ according to (N2) and the definition of $\oplus$. Hence $b c=0, a\left(b^{\prime} c^{\prime}\right)^{\prime}=0$ and $\left(a^{\prime} b^{\prime}\right) c^{\prime}=a^{\prime}\left(b^{\prime} c^{\prime}\right)$ according to (N4). Therefore $b \leq c^{\prime}$ and $a \leq b^{\prime} c^{\prime}$. This finally implies that there exist $b \oplus c$ and $a \oplus(b \oplus c)$ and

$$
(a \oplus b) \oplus c=\left(\left(a^{\prime} b^{\prime}\right) c^{\prime}\right)^{\prime}=\left(a^{\prime}\left(b^{\prime} c^{\prime}\right)\right)^{\prime}=a \oplus(b \oplus c) .
$$

(E3) If $a \oplus b$ exists and $a \oplus b=1$ then $a \leq b^{\prime}$ and $\left(a^{\prime} b^{\prime}\right)^{\prime}=1$ according to the definition of $\oplus$ and hence $a^{\prime} b^{\prime}=0$ by (N1), i.e. $a^{\prime} \leq b$ according to (N2) which together with $b \leq a^{\prime}$ implies $b=a^{\prime}$ according the antisymmetry of $\leq$. On the other hand, $a \leq a$ and $a^{\prime} \leq a^{\prime}$ and hence $a \oplus a^{\prime}$ exists and $a^{\prime} a=0$ according to the definition of $\oplus$ and (N2) and therefore $a \oplus a^{\prime}=\left(a^{\prime} a\right)^{\prime}=0^{\prime}=1$ according to (N1). Hence $a \oplus b=1$ if and only if $b=a^{\prime}$.

(E4) If $a \oplus 1$ exists then, using (N1), $a \leq 1^{\prime}=0$ and hence $a=0$.

Hence $\mathbb{E}(\mathbf{R})$ is an effect algebra. Let $\leq_{1}$ denote its induced order. According to Lemma 8 , $(R, \leq)=\left(R, \leq_{1}\right)$. Since the first poset is a lattice, the same is true for the second one.

Next we show that the described correspondence between lattice effect algebras and effect near semirings is one-to-one.

Theorem 10 Let $\mathbf{E}=\left(E, \oplus,{ }^{\prime}, 0,1\right)$ be a lattice effect algebra. Then $\mathbb{E}(\mathbb{R}(\mathbf{E}))=\mathbf{E}$.

Proof Let $\mathbb{R}(\mathbf{E})=\left(E, \vee, \cdot{ }^{\prime}, 0,1\right), \mathbb{E}(\mathbb{R}(\mathbf{E}))=\left(E, \oplus_{1},{ }^{\prime}, 0,1\right)$ and $a, b \in E$. Then the following are equivalent: $a \oplus_{1} b$ exists, $a \leq b^{\prime}, a \oplus b$ exists. If this is the case then $a \oplus_{1} b=$ $\left(a^{\prime} b^{\prime}\right)^{\prime}=\left(a \wedge b^{\prime}\right) \oplus b=a \oplus b$.

Theorem 11 Let $\mathbf{R}=\left(R,+, \cdot,{ }^{\prime}, 0,1\right)$ be an effect near semiring. Then $\mathbb{R}(\mathbb{E}(\mathbf{R}))=\mathbf{R}$.

Proof Let $\mathbb{E}(\mathbf{R})=\left(R, \oplus,{ }^{\prime}, 0,1\right), \mathbb{R}(\mathbb{E}(\mathbf{R}))=\left(R, \vee, \cdot_{1},{ }^{\prime}, 0,1\right), \leq$ and $\leq_{1}$ denote the induced orders of $\mathbf{R}$ and $\mathbb{E}(\mathbf{R})$, respectively, and $a, b \in R$. Then $\leq=\leq_{1}$ according to Lemma 8 and

$$
a \vee b=\sup _{\leq 1}(a, b)=\sup _{\leq}(a, b)=a+b .
$$

The last equality follows from the remarks in Definition 3. Moreover, $b^{\prime} b=0$ because of $b^{\prime} \leq b^{\prime}$ and, finally,

$a \cdot{ }_{1} b=\left(\left(a^{\prime} \wedge b\right) \oplus b^{\prime}\right)^{\prime}=\left(a^{\prime} \wedge b\right)^{\prime} b=\left(a \vee b^{\prime}\right) b=\left(a+b^{\prime}\right) b=a b+b^{\prime} b=a b+0=a b$.

Remark 12 Every effect near semiring $\mathbf{R}=\left(R,+, \cdot,{ }^{\prime}, 0,1\right)$ is congruence distributive since $\left(R,+,+^{\prime}\right)$ is a lattice and $+^{\prime}$ is a term function of $\mathbf{R}$. 
The representation of lattice effect algebras by means of effect near semirings enables us to classify some important classes of effect algebras. The following concept was introduced in [7].

Definition 13 A lattice orthoalgebra is a lattice effect algebra $\mathbf{E}=\left(E, \oplus,{ }^{\prime}, 0,1\right)$ satisfying condition (E5) for all $x \in E$ :

$$
\text { If } x \oplus x \text { exists then } x=0 \text {. }
$$

For the reader's convenience, we recall the following well-known concept.

An orthomodular lattice is an algebra $\mathbf{L}=\left(L, \vee, \wedge,{ }^{\prime}, 0,1\right)$ of type $(2,2,1,0,0)$ satisfying conditions $(\mathrm{O} 1)-(\mathrm{O} 4)$ :

(O1) $(L, \vee, \wedge, 0,1)$ is a bounded lattice;

(O2) the operation' is an antitone involution of $(L, \leq)$;

(O3) $x \vee x^{\prime} \approx 1$ and $x \wedge x^{\prime} \approx 0$;

(O4) $x \vee y \approx x \vee\left((x \vee y) \wedge x^{\prime}\right)$.

If $\mathbf{L}=\left(L, \vee, \wedge,{ }^{\prime}, 0,1\right)$ is an orthomodular lattice and $x \oplus y:=x \vee y$ if $x \leq y^{\prime}$ then $\mathbf{E}:=\left(L, \oplus,^{\prime}, 0,1\right)$ is a lattice orthoalgebra (cf. [7]). Conversely, the underlying lattice of a lattice orthoalgebra is orthomodular.

Now we can characterize lattice orthoalgebras by means of effect near semirings as follows:

Theorem 14 A lattice effect algebra $\mathbf{E}=\left(E, \oplus,{ }^{\prime}, 0,1\right)$ is a lattice orthoalgebra if and only if its corresponding effect near semiring $\mathbf{R}=\left(E,+, \cdot,{ }^{\prime}, 0,1\right)$ is multiplicatively idempotent, i.e. satisfies the identity $x x \approx x$.

Proof If $\mathbf{E}$ is a lattice orthoalgebra then $x \wedge x^{\prime} \approx 0$ (cf. Example 4.3 in [2]) and hence

$$
x x \approx\left(\left(x^{\prime} \wedge x\right) \oplus x^{\prime}\right)^{\prime} \approx\left(0 \oplus x^{\prime}\right)^{\prime} \approx\left(x^{\prime}\right)^{\prime} \approx x
$$

according to (F3) and (F4) and if, conversely, R satisfies $x x \approx x, a \in E$ and $a \oplus a$ exists then $a \leq a^{\prime}$ and hence $a=a a=a\left(a^{\prime}\right)^{\prime}=0$ according to (N2).

We can also characterize those lattice orthoalgebras whose underlying orthomodular lattice is a Boolean algebra (thus characterizing the classicality of the corresponding physical system). For this purpose we need some results on orthomodular lattices.

Recall that two elements $a, b$ of an orthomodular lattice $\left(L, \vee, \wedge,{ }^{\prime}, 0,1\right)$ are said to commute with each other ( $a \mathrm{C} b$, for short) if $a=(a \wedge b) \vee\left(a \wedge b^{\prime}\right)$. It is well-known that two comparable elements commute and that $a \mathrm{C} b$ implies $b \mathrm{C} a$ and $a \mathrm{C} b^{\prime}$. Also the following theorem is well-known:

Theorem 15 (Theorem of Foulis-Holland) If $\left(L, \vee, \wedge,{ }^{\prime}, 0,1\right)$ is an orthomodular lattice, $a, b, c \in L, a \mathrm{C} b$ and $a \mathrm{C} c$ then the sublattice of $(L, \vee, \wedge)$ generated by $\{a, b, c\}$ is distributive.

Now we are able to prove

Theorem 16 The underlying orthomodular lattice of a lattice orthoalgebra $\mathbf{E}=$ $\left(E, \oplus,{ }^{\prime}, 0,1\right)$ is a Boolean algebra if and only if its corresponding effect near semiring $\mathbf{R}=\left(R,+, \cdot,^{\prime}, 0,1\right)$ satisfies the identity $x+y \approx\left(x^{\prime} y^{\prime}\right)^{\prime}$. 
Proof Let $\mathbf{L}=\left(L, \vee, \wedge,{ }^{\prime}, 0,1\right)$ denote the orthomodular lattice corresponding to $\mathbf{E}$. If $\mathbf{L}$ is a Boolean algebra then

$\left(x^{\prime} y^{\prime}\right)^{\prime} \approx\left(\left(x^{\prime} \vee y\right) \wedge y^{\prime}\right)^{\prime} \approx\left(\left(x^{\prime} \wedge y^{\prime}\right) \vee\left(y \wedge y^{\prime}\right)\right)^{\prime} \approx\left(\left(x^{\prime} \wedge y^{\prime}\right) \vee 0\right)^{\prime} \approx\left(x^{\prime} \wedge y^{\prime}\right)^{\prime} \approx x \vee y \approx x+y$.

If, conversely, $\mathbf{R}$ satisfies $x+y \approx\left(x^{\prime} y^{\prime}\right)^{\prime}$ then

$$
x \wedge y \approx\left(x^{\prime} \vee y^{\prime}\right)^{\prime} \approx\left(x^{\prime}+y^{\prime}\right)^{\prime} \approx\left(\left(\left(x^{\prime}\right)^{\prime}\left(y^{\prime}\right)^{\prime}\right)^{\prime}\right)^{\prime} \approx x y \approx\left(x \vee y^{\prime}\right) \wedge y,
$$

$z^{\prime} \leq x \vee z^{\prime}$, and $z^{\prime} \leq y \vee z^{\prime}$ and hence $z^{\prime} \mathrm{C}\left(x \vee z^{\prime}\right)$ and $z^{\prime} \mathrm{C}\left(y \vee z^{\prime}\right)$ which implies $z \mathrm{C}\left(x \vee z^{\prime}\right)$ and $z \mathrm{C}\left(y \vee z^{\prime}\right)$ and therefore

$$
\begin{aligned}
(x \vee y) \wedge z & \approx\left((x \vee y) \vee z^{\prime}\right) \wedge z \approx\left(\left(x \vee z^{\prime}\right) \vee\left(y \vee z^{\prime}\right)\right) \wedge z \approx \\
& \approx\left(\left(x \vee z^{\prime}\right) \wedge z\right) \vee\left(\left(y \vee z^{\prime}\right) \wedge z\right) \approx(x \wedge z) \vee(y \wedge z),
\end{aligned}
$$

i.e., $\mathbf{L}$ is a Boolean algebra.

We recall that an $M V$-algebra is an algebra $\mathbf{M}=(M,+, \neg, 0)$ of type $(2,1,0)$ satisfying identities (M1) - (M6):

(M1) $x+y \approx y+x$

(M2) $\quad(x+y)+z \approx x+(y+z)$;

(M3) $x+0 \approx x$;

(M4) $\neg(\neg x) \approx x$;

(M5) $x+\neg 0 \approx \neg 0$;

(M6) $\neg(\neg x+y)+y \approx \neg(\neg y+x)+x$.

Lemma 17 If $\mathbf{M}=(M,+, \neg, 0)$ is an $M V$-algebra, $x \oplus y:=x+y$ if $\neg x+\neg y=1$ and $1:=\neg 0$ then $\mathbb{L}(\mathbf{M}):=(M, \oplus, \neg, 0,1)$ is a lattice effect algebra and $x+y=(x \wedge \neg y) \oplus y$ for all $x, y \in M$ (cf. [7] or Example 4.4 in [2]).

An interesting case of effect algebras are those which can be constructed as shown above. Hence we define

Definition 18 An $M V$-effect algebra (cf. [7]) is an effect algebra of the form $\mathbb{L}(\mathbf{M})$ with an MV-algebra M.

As already mentioned, Riečanová [9] showed that every lattice effect algebra is the settheoretic union of maximal subalgebras which are MV-effect algebras, so-called blocks, and therefore is itself an MV-effect algebra if and only if it consists of one block only. Recall from [2] the following statement:

Proposition 19 (cf. Theorem 4.6 in [2]) If $\mathbf{E}=\left(E, \oplus,{ }^{\prime}, 0,1\right)$ is a lattice effect algebra then $a, b \in E$ belong to the same block of $\mathbf{E}$ if and only if $a+b=b+a\left(\right.$ where $x+y:=\left(x \wedge y^{\prime}\right) \oplus y$ for all $x, y \in E$ ).

Now we can characterize MV-effect algebras by means of effect near semirings as follows:

Corollary 20 A lattice effect algebra $\mathbf{E}=\left(E, \oplus,{ }^{\prime}, 0,1\right)$ is an MV-effect algebra if and only if its corresponding effect near semiring $\mathbf{R}=\left(E,+, \cdot{ }^{\prime},{ }^{\prime}, 0,1\right)$ is commutative, i.e. satisfies the identity $x y \approx y x$. 
Proof If $\mathbf{E}$ is an MV-effect algebra then

$$
x y \approx\left(\left(x^{\prime} \wedge y\right) \oplus y^{\prime}\right)^{\prime} \approx\left(x^{\prime}+y^{\prime}\right)^{\prime} \approx\left(y^{\prime}+x^{\prime}\right)^{\prime} \approx\left(\left(y^{\prime} \wedge x\right) \oplus x^{\prime}\right)^{\prime} \approx y x
$$

and if, conversely, $x y \approx y x$ then

$$
x+y \approx\left(x \wedge y^{\prime}\right) \oplus y \approx\left(x^{\prime} y^{\prime}\right)^{\prime} \approx\left(y^{\prime} x^{\prime}\right)^{\prime} \approx\left(y \wedge x^{\prime}\right) \oplus x \approx y+x
$$

and hence $\mathbf{E}$ is an MV-effect algebra by Proposition 19 .

According to Proposition 19 and the proof of Corollary 20 two elements $a, b$ of a lattice effect algebra belong to the same block if and only if $a b=b a$ in its corresponding effect near semiring. Due to Riečanová's theorem (cf. [9]) we conclude

Corollary 21 Every effect near semiring is the set-theoretic union of commutative effect near semirings.

Acknowledgments Open access funding provided by Austrian Science Fund (FWF)

Open Access This article is distributed under the terms of the Creative Commons Attribution 4.0 International License (http://creativecommons.org/licenses/by/4.0/), which permits unrestricted use, distribution, and reproduction in any medium, provided you give appropriate credit to the original author(s) and the source, provide a link to the Creative Commons license, and indicate if changes were made.

\section{References}

1. Belluce, L.P., Di Nola, A., Ferraioli, A.R.: MV-semirings and their sheaf representations. Order 30, 165179 (2013)

2. Chajda, I., Halaš, R., Kühr, J.: Many-valued quantum algebras. Algebra Universalis 60, 63-90 (2009)

3. Chajda, I., Länger, H.: A representation of basic algebras by coupled right near semirings. Acta Sci. Math. (Szeged) 81, 361-374 (2015)

4. Chajda, I., Länger, H.: Coupled right orthosemirings induced by orthomodular lattices. Order. doi: 10.1007/s11083-015-9383-7

5. Chajda, I., Länger, H.: A triple representation of lattice effect algebras. Math. Slovaca (to appear)

6. Di Nola, A., Russo, C.: The semiring-theoretic approach to MV-algebras: A survey. Fuzzy Sets Syst. 281, 134-154 (2015)

7. Dvurečenskij, A., Pulmannová, S.: New Trends in Quantum Structures. Kluwer, Dordrecht (2000). ISBN 0-7923-6471-6

8. Foulis, D.J., Bennett, M.K.: Effect algebras and unsharp quantum logics. Found. Phys. 24, 1331-1352 (1994)

9. Riečanová, Z.: Generalization of blocks for D-lattices and lattice-ordered effect algebras. Int. J. Theor. Phys. 39, 231-237 (2000) 